\title{
Changes in the physical fitness of elite women's rugby union players over a competition season
}

\author{
N M Hene, MA (Sport Science); S H Bassett, BSc (Hons) (Biokinetics), MSc (Sports Science), PhD \\ Department of Sport, Recreation and Exercise Science, University of the Western Cape, Bellville, South Africa
}

Corresponding author: N M Hene (ncebahene@hotmail.com)

\begin{abstract}
Objective. To investigate changes in the physical fitness characteristics of elite women's rugby union players over a competitive season. Methods. Thirty-two elite women's rugby union players, all members of the South African Rugby Union High Performance Squad, were sub-divided into 2 positional categories of 17 forwards and 15 backs, respectively, and assessed pre-, mid- and post-competition season. Players underwent anthropometric (stature, body mass and sum of 7 skinfolds) and physical performance measurements (vertical jump, $10 \mathrm{~m}$ and $40 \mathrm{~m}$ sprint, 1 repetition maximum (1RM) bench press and multi-stage shuttle-run test).

Analysis. A 2-factor analysis of variance was used to evaluate differences in physical fitness variables between and within playing positions over the competition season $(p<0.01)$.

Results. In both groups, no significant changes were detected in the sum of skinfolds, vertical jump height, 1RM bench press and multi-stage shuttle-run test scores throughout the season. However, sprint times (10 $\mathrm{m}$ and $40 \mathrm{~m}$ ) significantly increased and then decreased for both groups between the early (pre- to mid-season) and later phases of the season (mid- to post-season), respectively.

Conclusion. The results suggest that, for improvement in physical fitness, players need to train at higher loads, especially in the preparatory phase. Thereafter, they must take measures to actively maintain these gains throughout the competitive season. Direct supervision of their conditioning should be encouraged.
\end{abstract}

S Afr J SM 2013;25(2):47-50. DOI:10.7196/SAJSM.371

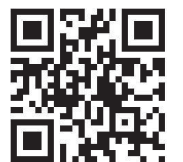

The role of women within the rugby sphere has traditionally been one of provision rather than active participant. ${ }^{[1]}$ Over the past 2 decades, rugby union has grown unexpectedly as a female participative sport in Australia, Great Britain, Canada, the USA, New Zealand and many other Western and Asian countries. ${ }^{[2]}$ In 2000, when the South African Rugby Union (SARU) accepted women's rugby into the rugby fraternity, there were $<10$ clubs playing on a social basis. This inclusion culminated in the Springbok Women's Team participating in the 2006 International Rugby Board Women's World Cup in Canada. ${ }^{[3]}$

Rugby union is a contact sport in which players require high levels of physical fitness, composite of aerobic fitness and anaerobic endurance, muscle strength and power, speed, agility and body composition. ${ }^{[4]}$ The seasonal nature of rugby imposes varied physical stresses that may compromise physical development. ${ }^{[5]}$ Hence, the capacity of elite players to acquire and maintain good physical fitness pre- and in-season, has become paramount. ${ }^{[6]}$ Male rugby players show the greatest improvement in strength, flexibility, aerobic fitness and reduction in skinfold thickness pre-season, before returning to baseline or maintaining values during a competitive season. ${ }^{[7-9]} \mathrm{A}$ reduction in muscular power, maximal aerobic fitness and increased skinfold thickness may also occur towards the end of the rugby season, due to low training loads, high match loads and injury rate ${ }^{[8]}$ However, little is known about the changes in physical fitness characteristics of women rugby players over a competitive season.

Several studies have described the anthropometric and physical performance characteristics of women's rugby players; however, these studies were limited to a single fitness-testing session performed either pre-, mid- or post-season. ${ }^{[4,10-12]}$ Studies investigating changes in the physical fitness characteristics of women during a season for soccer, ${ }^{[13]}$ handball ${ }^{[14]}$ and hockey, ${ }^{[15]}$ have reported desirable changes in body composition (a reduction in percentage body fat or skinfold thickness and an increase in lean muscle mass) and minimal change in body composition pre- ${ }^{[13,14]}$ and in-season, ${ }^{[15]}$ respectively. However, no stated changes in aerobic fitness ${ }^{[15]}$ and muscle strength ${ }^{[16]}$ were reported over the entire season.

To date, no published study has monitored the physical fitness levels of women rugby union players over the course of a competition season; hence, the purpose of our study. This is important because the primary goal of pre-season training is to optimise fitness and enhance performance during in-season competition. ${ }^{[14]}$ We hypothesised that the physical fitness characteristics of elite women's rugby players would significantly improve from pre- to mid-season, and be maintained during the season, as is seen in men's rugby. ${ }^{[17,18]}$

\section{Methods}

Research design and sample

Thirty-two women rugby players (mean age 27 years; standard deviation (SD) \pm 4 ; range 19 - 17), who were selected for the SARU High Performance Squad of the 2010 Women's Rugby World Cup, were recruited for the study. Players were selected from the 2008 interprovincial competition by national selectors appointed by the SARU. The players were grouped according to forward (5 props, 2 hookers, 4 locks and 6 loose forwards) and back-line players ( 9 inside backs and 
6 outside backs). Prior to fitness testing, all players provided informed consent to study participation. Ethical clearance to conduct the study was obtained from the University of the Western Cape.

\section{Testing procedure}

Anthropometric and physical performance measurements were conducted on 3 occasions during a 32-week season (April - November 2009), which encompassed pre- (weeks 1 - 12) and in-season periods (weeks 13 - 32), provincial games, and a 4-week international tour. The first test (T1) (May 2009) was performed 2 weeks after the start of pre-season; the second test (T2) (July 2009) in the middle of the competition season; and the third test (T3) (November 2009) 2 weeks after the end of the interprovincial league finals.

Players followed an individualised, but unsupervised, prescribed strength-and-conditioning programme, both pre- and in-season. Pre-season, the programme required 3 resistance-training sessions and 2 high-intensity running sessions per week. In-season training was reduced to 1 - 2 resistance-training sessions and 1 high-intensity running session per week, plus match play.

Unfortunately, timing of the testing sessions could not be controlled and was not evenly distributed throughout the season, but rather scheduled around players' work, study and playing commitments. Physical fitness testing coincided with national training camps and all players were assessed on the first day of each camp by the sports physician and physiotherapist, who conducted a full medical examination and musculoskeletal screening. Players with a medical condition or injury were excluded from the physical fitness assessment. Although many physical attributes are required for successful performance in rugby union, only selected anthropometric and physical fitness tests were investigated, due to time constraints and player workload during the training camps (e.g. agility and anaerobic capacity were not assessed). Similarly, the researchers did not assess lower-body strength due to the lack of identical strengthtesting equipment at all camps.

The fitness-testing protocol was as follows: body mass was measured using a calibrated Seca balance beam ( $0.1 \mathrm{~kg}$ accuracy); skinfold thickness was then measured at 7 sites (biceps, triceps, subscapular, suprailiac, medial calf, front thigh and abdominal) with Harpenden skinfold callipers; ${ }^{[15]}$ explosive leg power was tested using the Vertec Jump Tester (Sports Imports, Columbus, USA) and standard protocol; $;^{[16]} 10 \mathrm{~m}$ and $40 \mathrm{~m}$ sprinting speed was evaluated with an electronic sprint timer with photo-electric sensors; ${ }^{[19,20]}$ upper-body strength was evaluated with a 1 repetition maximum (1RM) bench press test (the final weight (in $\mathrm{kg}$ ) successfully lifted was recorded as the absolute $1 \mathrm{RM}) ;^{[21]}$ and finally, the progressive multi-stage shuttle-run test was conducted ${ }^{[22]}$ (with the final shuttle achieved recorded as the aerobic fitness score).

\section{Statistical analysis}

Descriptive statistics were used to present the results. Changes in physical fitness characteristics at the 3 time-points in the season between playing positions were compared using a 2 -factor analysis of variance (ANOVA), with the between-participants factor (player position) having 2 levels and the within-participants factor (time of testing) having 3 levels. The responses within participants were dependent; dependency was modelled using an unstructured correlation model. An interaction term for the 2 factors was included in the model. Pairwise comparisons were done using least-squares means. Due to the large number of tests conducted, a more stringent level of significance of $p<0.01$ was used rather than $p<0.05$.

\section{Results}

\section{Body mass, skinfold thickness, muscular strength and power}

There was a significant decrease $(p=0.007)$ in body mass among the backs from pre- to mid-season; no changes were observed from midto post-season. There were no significant changes in body mass among the forwards throughout the season. Over the 3 testing sessions, there were no significant changes in skinfold thickness among the backs; however, the sum of skinfolds in the forwards decreased significantly from pre- to post-season $(p=0.001)$. No significant differences were noted for explosive leg power and muscular strength in either group.

\section{Speed}

The times for the $10 \mathrm{~m}$ sprint, for both forwards and backs, were significantly slower $(p<0.001)$ from pre- to mid-season, but improved significantly from mid- to post-season $(p<0.001)$. Furthermore, the forwards achieved significantly faster $10 \mathrm{~m}$ times post-season compared with pre-season $(p<0.000)$, whereas there was no significant change in the backs' sprint times over the same period. The backs achieved significantly faster times from pre- to mid-season in the $40 \mathrm{~m}$ sprint $(p<0.000)$, whereas the forwards' speed did not change significantly during this period. However, from mid- to postseason, as well as from pre- to post-season, both groups' times were significantly faster $(p<0.001)$.

\section{Aerobic fitness}

An assessment of the forwards' aerobic fitness over the course of the season (multi-stage shuttle run), showed a significant decrease in the number of shuttles successfully completed from pre- to midseason ( $p=0.009$ ), but a significant increase from mid- to post-season $(p=0.001)$, with no significant change over the season. In contrast, no significant differences were observed among the backs.

\section{Discussion}

In male rugby players, desirable changes in body composition (decrease in skinfold thickness) have been demonstrated pre-season when training volume was high. ${ }^{[5,-9]}$ Furthermore, skinfold thickness was shown to be maintained throughout the competitive phase of the season when training loads were reduced, and match loads and injuries were at their highest. ${ }^{[7,10]}$ There was no significant decrease in body mass, yet there was a significant decrease in skinfolds noted for the forwards in our study, contradictory to the results found among males. For the backs, there was a significant increase in weight from pre- to mid-season, with no significant change post-season and no significant change in the sum of skinfolds throughout the season. These results may reflect that women rugby players do not come into the pre-season at the optimal weight in the first place, with adaptations taking place to the training and match requirements at the beginning of the season. Perhaps the training volume, intensity and/or frequency to elicit improvements in body-fat levels among the elite women's rugby players prior to the season may have been inappropriate, or the players were simply not adhering to their preseason training programme as strictly as requested. 


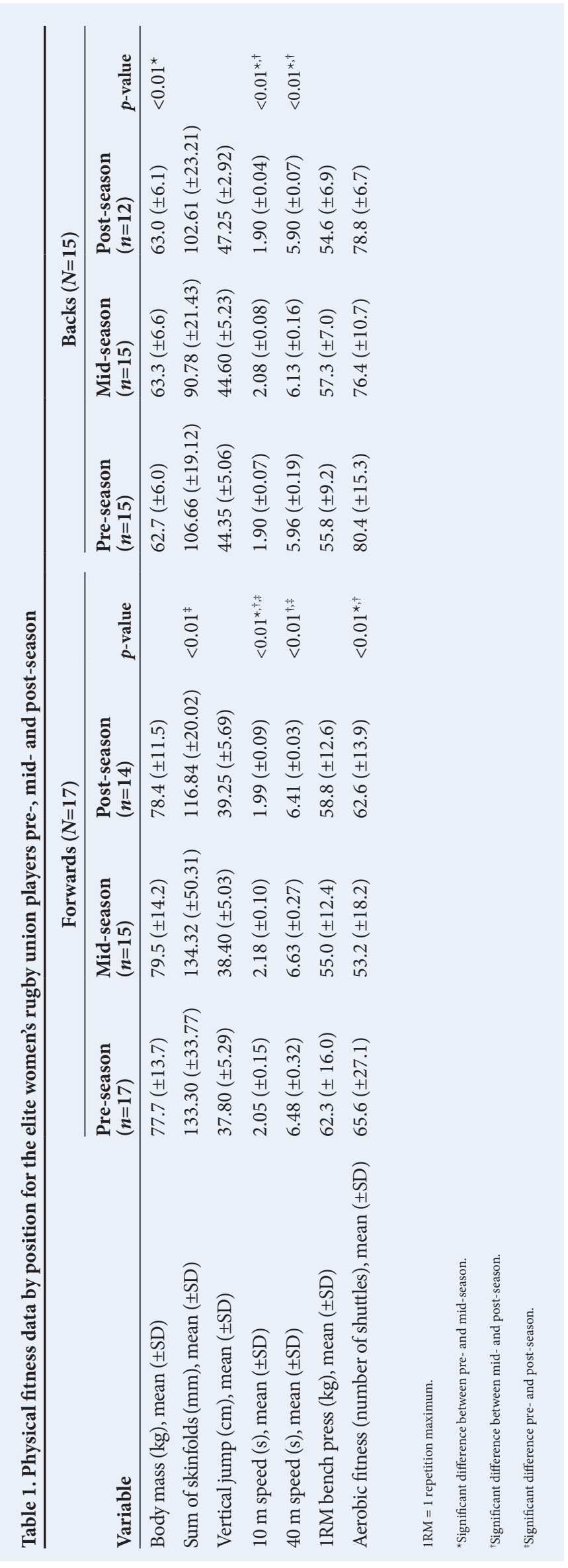

The results obtained from both groups show that there were no changes in vertical jump performance over the season. The relatively modest change in jumping height within the participants could perhaps have been attributed to a greater focus on gym-based resistance training inseason, as well as a lack of plyometric training throughout the season. Hoff et al. ${ }^{[23]}$ suggested that in order to enhance vertical jumping ability, a well-designed training programme that includes short plyometric sessions should be implemented as part of the strengthand-conditioning programme.

The sprint times for all players over $10 \mathrm{~m}$ and $40 \mathrm{~m}$ were contrary to previous research on senior ${ }^{[17]}$ and junior ${ }^{[8]}$ rugby league players, where times remained relatively unchanged throughout the season. The significant decrease in sprint performance noted for the backs between pre- and mid-season was most likely due to the significant increase in body mass during this time. Although not significant, the forwards also increased in body weight during this time, which could have translated into their increased sprint times mid-season. This decrease in sprint times in the early stages of the season is of concern, because speed is an important indicator of a player's athletic ability. ${ }^{[21]}$ Further research on the effects of concurrent training on the maintenance or improvement of speed in female rugby players would increase our understanding of the changes in physical fitness during the preparation phase.

The goal of all pre- and in-season conditioning programmes for rugby players should be to maximise muscular fitness before the season and maintain the pre-season gains, respectively. ${ }^{[24]}$ In this study, the 1RM bench press remained constant for backs and forwards pre- and in-season, despite the players being prescribed a periodised strength-and-conditioning programme during this time. It has been suggested that a lack of strength gains in professional athletes is likely due to concurrent training ${ }^{[24]}$ and strength-training, ${ }^{[24,26]}$ which may reduce the scope for further improvements. Although this study did not research the training programme per se, the lack of improvement in upper-body strength results may have been due to a lack of direct supervision by a strength coach and/or possible insufficient training frequency and volume required to increase upper-body strength. This notion is supported by Coutts et al., ${ }^{[27]}$ who noted that direct supervision of resistance training resulted in increased strength gains compared with unsupervised training.

A number of studies of male rugby players have reported an increase in aerobic fitness during the preparatory phase of the season, ${ }^{[5,9,17]}$ with a decrease observed towards the end of the season. ${ }^{[17]}$ The significant improvements in fitness in the early stages of the season have been attributed to the high training loads experience during this period, ${ }^{[17]}$ while reductions in aerobic fitness as the season progresses may be due to lower training loads and higher match loads and injury rates during this period. ${ }^{[28]}$ In contrast to these findings, the current study showed no changes in aerobic fitness for backs over the entire season; however, the forwards followed a similar trend as seen in their male counterparts. ${ }^{[5,9,17]}$ This lack of improvement in aerobic fitness during the preparatory phase in elite women's rugby players may also be an indication of the lack of daily supervised training, which would otherwise result in greater training adherence and intensity. ${ }^{[25]}$

Previous studies of the physical fitness characteristics of women's rugby players have been limited to a single fitness test performed during a competitive season. ${ }^{[4,10-12]}$ Our study is the first to document 
changes in physical fitness characteristics of women's rugby players over the duration of a competitive season. This study has highlighted the lack of fitness in general in the South African women's rugby team and the absence of improvement over the season that would be expected of a men's side.

\section{Conclusion}

Our study revealed no significant improvements in skinfold thickness, explosive leg power, upper-body muscular strength and aerobic fitness within forwards and backs between pre- and mid-season. This suggests that the conditioning programme of the players throughout the season was inappropriate, or that their adherence to the prescribed programme was inadequate, or both. It is suspected that female players do not adhere to unsupervised training programmes; direct daily training supervision, similar to that of their male counterparts, is recommended, and strength-and-conditioning coaches should be more directly involved with player preparation. This is not an easy task, as female rugby players are currently not exposed to the same organised club, league, training structures, etc. as their male counterparts, and do not have access to similar infrastructure, especially well-equipped training facilities. To improve the physical fitness of women rugby players, we suggest the implementation of supervised strength-and-conditioning sessions. However, further studies are required to determine the appropriate training stimulus thus required.

Coaches need to be cognizant of this general lack of fitness, which increases the risk of injury. Substantial resources and emphasis should be directed towards women's rugby players achieving pre-determined fitness levels at various age categories, as with the men's game, to allow more effective toleration of the physical demands of competition. However, this is a double-edged sword, as there are perhaps not enough women playing the game at this point to be so selective.

We recommend that existing men's rugby structures should assist the women's game more, especially as Rugby Sevens will debut at the 2016 Olympic Games. As women's rugby is currently developing rapidly the world over, South Africa must look at these issues in order to compete internationally. Lastly, much more research needs to be conducted on this form of the game.

\section{References}

1. Haynes J, Miller J. Women playing rugby, an 'old boys game': Beyond the social expectations. http://www.aare.edu.au/01pap/mil01183.htm (accessed 12 October 2009).

2. Chu MML, Leberman SI, Howe BL, Bachor DG. The black ferns: The experiences of New Zealand's elite women rugby players. J Sport Behav 2003;26(2):109.

3. International Rugby Board. SA Rugby Union. http://www.irb.com/unions/ union $=11000034 /$ index.html (accessed 12 October 2009).

4. Hene NM, Bassett SH, Andrews BS. Physical fitness of elite women's rugby union players. Afr J Phys Health Educ Recr Dance 2011;17(Suppl 1):1-8.

5. Holmyard DJ, Hazeldine RJ. Seasonal Variations in the Anthropometric and Physiological Characteristics of International Rugby Union Players. In: Reilly T, ed. Science and Football. London: E \& FN Spon, 1993:21-26.

6. Caldwell BP, Peters DM. Seasonal variation in physiological fitness of a semiprofessional soccer team. J Strength Cond Res 2009; 25(5):1370-1377. [http://dx.doi. org/10.1519/JSC.0b013e3181a4e82f]
7. Duthie GM, Pyne DB, Hopkins WG, Livingstone S, Hooper SL. Anthropometry profiles of elite rugby players: Quantifying changes in lean mass. Br J Sports Med 2006;40(3):202-207. [http://dx.doi.org/10.1136/bjsm.2005.019695]

8. Gabbett TJ. Physiological and anthropometric characteristics of junior rugby league players over a competitive season. J Strength Cond Res 2005;19(4):764-771. [http:// dx.doi.org/10.1519/00124278-200511000-00007]

9. Tong RJ, Mayes R. The Effect of Pre-season Training on the Physiological Characteristics of International Rugby Union Players. In Reilly T, Bangsbo J, Hughes M, eds. Science and Football III: Proceedings of the Third World Congress of Science and Football. London: E \& FN Spon, 1995:92-102.

10. Gabbett TJ. Physiological and anthropometric characteristics of elite women rugby league players. J Strength Cond Res 2007;21(3):875-881. [http://dx.doi. org/10.1519/00124278-200708000-00038]

11. Kirby WJ, Reilly T. Anthropometric and Fitness Profiles of Elite Female Rugby Union Players. In: Reilly T, ed. Science and Football. London: E \& FN Spon, 1993:62-72.

12. Quarrie KL, Handcock P, Waller AE, et al. The New Zealand rugby injury and performance project. III. Anthropometric and physical performance characteristics of players. Br J Sports Med 1995;29(4):263-270. [http://dx.doi.org/10.1136/ bjsm.29.4.263]

13. Clark M, Reed DB, Crouse SF, Armstrong RB. Pre- and Post-Season dietary intake, body composition and performance indices of NCAA Division 1 female soccer players. Int J Sport Nutr Exerc Metab 2003;13(3):303-319.

14. Granados C, Izquierdo M, Ibáñez J, Ruesta M, Gorostiaga EM. Effects of an entire season on physical fitness in elite female handball players. Med Sci Sports Exerc 2008;40(2):351-361. [http://dx.doi.org/10.1249/mss.0b013e31815b4905]

15. Astorino TA, Tam PA, Rietschel JC, Johnson SM, Freedman TP. Changes in physical fitness parameters during a competitive hockey season. J Strength Cond Res 2004;18(5):850-854. [http://dx.doi.org/10.1519/00124278-200411000-00029]

16. Ross WD, Marfell-Jones MJ. Kinanthropometry. In: MacDougall JD, Wenger HA, Green HJ, eds. Physiological Testing of the High-Performance Athlete. Champaign, IL: Human Kinetics, 1991:223-308.

17. Gabbett TJ. Changes in physiological and anthropometric characteristics of rugby league players during a competitive season. J Strength Cond Res 2005;19(2):400-408. [http://dx.doi.org/10.1519/00124278-200505000-00027]

18. Gabbett TJ, King T, Jenkins D. Applied physiology of rugby league. Sports Med 2008; 38(2):119-138. [http://dx.doi.org/10.2165/00007256-200838020-00003]

19. Baechle TR, Earle RW, Wathan W. Resistance training. In: Baechle TR, Earle RW, eds. Essentials of Strength Training and Conditioning. Champaign, IL: Human Kinetics, 2000:381-412.

20. Durandt J, du Toit S, Borrensen J, et al. Fitness and body composition profiling of elite junior South African rugby players. South African Journal of Sports Medicine 2006;10(3):38-45.

21. Logan P, Fornasiero D, Abernethy P, Lynch K. Protocols for the Assessment of Isoinertial Strength. In: Gore CJ, ed. Physiological Tests for Elite Athletes. Champaign, IL: Human Kinetics, 2000:200-221.

22. Léger LA, Lambert J. A maximal multistage $20 \mathrm{~m}$ shuttle run test to predict $\mathrm{VO}_{2}$ max. Eur J Appl Physiol Occ Phys 1982;49:1-2. [http://dx.doi.org/10.1007/BF00428958]

23. Hoff J, Kemi OJ, Helgerud J. Strength and endurance differences between elite and junior elite hockey players: Allometric scaling of strength measurement to body size. Eur J Appl Physiol 2005;102:739-745.

24. Hrysomallis C. Upper body strength and power changes during a football season. J Strength Cond Res 2010;24(2):557-559. [http://dx.doi.org/10.1519/ JSC.0b013e3181c09c9c]

25. Kraemer WJ, French DN, Paxton NJ, et al. Changes in exercise performance and hormonal concentrations over a big ten soccer season in starters and nonstarters. J Strength Cond Res 2004;18(1):121-128. [http://dx.doi.org/10.1519/00124278200402000-00018]

26. Baker D. The effects of an in-season of concurrent training on the maintenance of maximal strength and power in professional and college-aged rugby league football players. J Strength Cond Res 2001;15(2):172-177. [http://dx.doi. org/10.1519/00124278-200105000-00004]

27. Coutts AJ, Murphy AJ, Dascombe BJ. Effect of direct supervision of a strength coach on measures of muscular strength and power in young rugby league players. J Strength Cond Res 2004;18(2):316-323. [http://dx.doi.org/10.1519/00124278200405000-00021]

28. Gabbett TJ, Kelly JN, Sheppard JM. Speed, change of direction speed, and reactive agility of rugby league players. J Strength Cond Res 2008;22(1):174-181. [http:// dx.doi.org/10.1519/JSC.0b013e31815ef700] 